\title{
The Effect of Mandalynth Tracing as a Mindfulness- Based Training Technique on Anxiety and Self- Perception of Ability to Participate in Meaningful Activities
}

\author{
Christine Manville*, Thu Can, Leah Carlisle, Sarah Glassner, Caroline Goffigon, Hope Lohmueller and Jenae \\ Stevens
}

Department of Psychology, Belmont University, USA.

*Corresponding author: Christine Manville, Department of Psychology, Belmont University, USA.
Received Date: October 05, 2019

Published Date: December 09, 2019

\begin{abstract}
This study examined the impact of Mandalynth tracing as a mindfulness-based training technique. It investigated whether the act of tracing had an influence on an individual's physiology and self-perception of anxiety, and their perceived ability to participate in meaningful activities. This descriptive, mixed-methods study used interview responses, the Beck Anxiety Inventory, and pulse-oximeter readings to obtain data. While this study was not statistically significant, researchers concluded anxiety has a negative impact on individual perception of participation. Though additional research is needed to confirm the efficacy of Mandalynth tracing, the use of this tool decreased physiological symptoms of anxiety and promoted self-reported relaxation and calming effects.
\end{abstract}

\section{Introduction}

According to the World Health Organization (WHO) [1], mental health is defined as, "a state of well-being in which each individual realizes his or her own potential, can cope with the normal stresses of life, can work productively and fruitfully, and is able to make a contribution to her or his community". By this definition, mental health can be understood as a continuum. An individual's specific location on this continuum can fluctuate depending on a variety of internal and external variables, which can include stressors, anxiety, or physiological changes. The WHO has identified mental health as "a growing cause of disability worldwide and predicts that in the future, mental illness...will be the top cause of disability".

According to the National Alliance on Mental Illness (NAMI) [2], one in five adults in the United States experiences mental health issues in a given year. Individuals living with serious mental health issues have an increased risk of chronic medical conditions and live an average of twenty-five years less than other Americans [2]. Anxiety is a common co-occurring symptom with mental health disorders and has both mental and physical implications for an individual [3-5]. Symptoms of anxiety can interfere with one's ability to engage in a range of chosen occupations and affects perception of abilities [6,7]. Research has revealed a variety of techniques that can be used to help individuals cope with anxiety and stress [8]. Among the professions who work toward this goal with clients are occupational therapists. The goal of occupational therapy is to empower others to "achieve health, well-being, and participation in life" [9] through engagement in occupations. Activities and strategies which reduce symptoms of mental illness and promote participation can potentially reduce anxiety and enable individuals to have a better quality of life.

Complementary and Alternative Medicines (CAMs), among many other interventions, have promising outcomes for decreasing symptoms of mental illness [10]. CAMs are defined as "a group of diverse medical and health care systems, practices, and products that are not presently considered to be part of conventional medicine" [11]. A key responsibility of occupational therapy practitioners is to teach clients who are experiencing mental illness "strategies for managing symptoms to enhance occupational 
performance" [6], of which CAMs can play an important role [12]. The CAMs currently being used include guided imagery, massage, meditation, yoga, behavioral relaxation training, and mindfulness practices.

One component of CAMs relates to channeling mindfulness Mindfulness is described as a "purposeful, non-anxious, reflective practice" [13] and can be a means to gain self-acceptance and cope with anxiety, stress, anger, and other negative emotions [14]. Mindfulness-based interventions have been shown to benefit individuals with a variety of mental health issues, including anxiety, depression, and schizophrenia spectrum disorders [15]. These interventions have also been shown to boost mood [16], increase one's sense of well-being [17], and reduce perceived stress and rumination [18].

Research suggests mindfulness techniques can be effective in empowering adolescents with mental health issues to self-soothe and relieve emotional distress [19]. Mindfulness techniques encourage focused attention on the present moment, which has been shown to reduce symptoms of anxiety and mental illness [20]. Tracing and drawing are considered mindfulness techniques because they have calming effects shown to decrease an individual's perceived anxiety and stress. Mandalynth tracing is a similar kinetic activity, involving the use of a continuous pattern to evoke a flow of uninterrupted movement. There is a need for evidence concerning the use of Mandalynth tracing and its effects on anxiety.

Utilizing Mandalynth tracing as a mindfulness-based tool, this study investigated the influence of Celtic Art Mandalynths on anxiety levels and perception of participation through changes in pulse rate, oxygen saturation, and qualitative interviews. The Mandalynths used in this study were created by artist Erin Rado, who states that Mandalynth tracing simulates mindfulness through focus on intricate patterns (2016). The Mandalynth designs selected for this study were inspired by Celtic knots, and consisted of an assortment of continuous patterns, which varied in difficulty level based on the tightness or openness of the loops.

\section{Methods}

All participants in this mixed-methods study were receiving residential services in the local community through Park Center [21], a local community agency which has been providing services to individuals in recovery for over thirty years. In order to qualify for these services, individuals must have met the following criteria:

- $\quad$ be between the ages of 18 and 24 ,

- have a documented diagnosis of severe and persistent mental illness, and

- have a history of mental health hospitalization or adolescent foster care, and/or were currently experiencing homelessness, and

- the participants could not be a registered sex offender or have a history of acting out with sexual behaviors.
Ethical approval for this study was granted by an Institutional Review Board (IRB). All residents were invited to an initial meeting to discuss the research plan, their possible participation in this study, and any additional questions or concerns they may have. Individuals who wished to participate in the study signed a consent form and completed a demographic form. In total, seven residents chose to participate in the study; however, one participant dropped out of the study after the first session due to an exacerbation of psychotic symptoms.

\section{Structure and Procedures}

Researchers met with participants for thirty minutes once a week for four sessions. During these sessions, a pre-tracing pulse oximeter measurement was recorded in order to establish a baseline for each participant's heart rate and oxygen saturation levels. The participants selected a Mandalynth design from one of eight different potential patterns and then spent five minutes tracing with their index finger on their dominant hand. Post tracing, a second measurement was taken with the pulse oximeter, recording their heart rate and oxygen saturation level. At the end of each session, participants engaged in a six-question interview about their feelings and thoughts before and during the tracing and their self-perception of ability to perform meaningful activities. During the first and fourth week, the participants also completed a written Beck Anxiety Inventory (BAI). In the final session, participants completed six additional questions encouraging reflection on selfperception of anxiety and how they felt their anxiety changed over time, if at all.

\section{Evaluation Procedures}

The BAI is a twenty-one item self-report measure in which participants rate their perceived levels of anxiety. The BAI was used twice, once before the study began to establish a baseline level of anxiety and again at the end of the study to allow for comparison to see if any changes had occurred. The BAI uses a Likert scale for self-report, rating how often they experienced common physical symptoms of anxiety, such as: tingling, trembling hands, sweating or an inability to relax. Beck, et al. [22] report the test-retest reliability for the BAI as 0.75. The BAI scale was selected because of its simple rating system and non-leading questions, which facilitate unbiased responses to feelings and thoughts about anxiety.

The interview questions were created using a triangulation method. The content validity of the questionnaire was enhanced by drawing information from a review of the literature on mindfulness, participation, quality of life, and anxiety. The questions were reviewed by administrators from the residential program and a licensed occupational therapy practitioner, with ten years of experience in mental health. To provide face validity, interview questions were pilot- tested by members of another program who were not research participants who also had a diagnosis of mental illness.

Researchers measured physiological symptoms of anxiety using a pulse oximeter. In stressful situations, the body releases 
the hormone adrenaline, which will bring about an increase in heart rate and blood pressure [23]. Heart rate and oxygen saturation were measured before and after each session to evaluate whether Mandalynth tracing had an effect on these physiological manifestations of anxiety.

\section{Analysis of the Data}

Individual structured pre and post interviews with participants were transcribed, and the data collected during each interview was analyzed using the coding system NVivo. In order to increase validity, multiple researchers worked to code the data and compare results, looking for emerging themes and patterns.

Both parametric and non-parametric methods were used to analyze the quantitative data. Measures of central tendency, variability, and dispersion were calculated for ordinal data in the interview questions as well as pre and post oxygen saturation and heart rate levels. This data was then analyzed using the Friedman Test. This test was used because of the study's small sample size and to compare changes in the participant's' physiological symptoms week to week. The BAI was analyzed using the Wilcoxon Two-Tailed Signed Rank Test, to compare participant's anxiety levels at the beginning and end of the study.

\section{Results}

The physiological results of pre- and post-tracing were not statistically significant; however, there were trends in the data. The lines depicting each individual's pre- and post-session pulse values are portrayed. While median values for the group decreased during weeks one (-4.5), three (-7.0), and four (4.0), the difference in the amount of change among the four sessions was not statistically significant (Friedman test, $\mathrm{X} 2=3.24, \mathrm{p}=0.356$ ). The Friedman test was used for the nonparametric data to analyze the difference between the pre- and post-test for each of the weeks for individual participants. Changes in oxygen saturation levels within sessions are displayed similarly. Minimal changes were detected in any session (median values: week 1=-1.0, week $2=-1.0$, week $3=1.0$ week $4=0.0$ ) and the difference in the amount of change among the four sessions was not statistically significant (Friedman test, X2 = $5.86, p=0.119$ ). During the four weeks of research, the majority of the participants' pulse and oxygen saturation levels decreased after the Mandalynth tracing was completed.

Due to small sample size and variable physiological information, qualitative data is more revealing of the participants' lived experience. Self-report data from the Beck Anxiety Inventory generally followed a similar pattern to the physiological data. The median change in Beck score from session one to session four was -2.0 yet was not statistically significant (Wilcoxon Signed-Rank test, $\mathrm{z}=1.21, \mathrm{p}=0.225$ ). The Wilcoxon Signed-Rank test was used to analyze the difference between the nonparametric pre- and posttests between the participants. As shown, five of the six participants displayed some level of decrease in self-reported anxiety over the study period. Based on the results of the BAI, there was a general trend of decreased anxiety over time.
Answers from interview questions were coded in NVivo. The most commonly used words from the interview responses were "good," "calm," and "blank." Half of the participants experienced a positive or calming change in thoughts and feelings while completing the Mandalynth tracing, and reported feeling "relaxed," or "focused." Some participants fluctuated between feeling calm and having no effect at all. During one particular session, participant six felt, "nervous anxious trying to do it fast enough".

During the final interview, the participants were asked what aspects of the Mandalynths were meaningful or helpful to them personally. Four of the six participants reported the Mandalynth tracing had calming, relaxing, and focusing effects. Participant four used Mandalynth tracing as a coping strategy on a daily basis and stated, "When I started to get frustrated, I traced engravings in a table at work when I didn't have a Mandalynth." One of the participants noted the Mandalynth tracing was only helpful for approximately an hour, and another participant did not feel the tracing was helpful at all.

\section{Discussion}

Despite results indicating lack of statistical significance in the decrease of oxygen saturation levels and heart rate, the majority of participants experienced an overall decrease in both, suggesting that Mandalynth tracing has an effect on lowering anxiety levels. In contrast, participant six displayed an increased heart rate after the Mandalynth tracing, stating they felt, "anxious [because they were] rushing through, trying to compete against [themselves]." This experience indicated tracing can also be a source of anxiety if a person perceives it as a contest. Another participant, number four, experienced psychosis in week three of the study, which increased the participant's heart rate and oxygen saturation levels. In week one of the study, this participant chose a tight Celtic knot for his tracing. When this individual began to display heavy breathing and frustration, a less complex knot was presented. This individual's expressed frustration suggests the complexity of the knots can also influence anxiety levels. The experiences of participants four and six show while Mandalynth tracing may be a beneficial strategy for some to incorporate, it may not be appropriate for all individuals. Personal preferences and present symptoms of mental illness should be considered when recommending coping strategies.

Information gathered from interviews show the participants believed their emotions and anxiety affected their participation in occupation. For example, one participant stated, "[participation is affected] depending on my mood [when I'm] depressed or angry it is detrimental to work [when I'm in] a good mood I focus on tasks and am more goal oriented." With this in mind, coping strategies, which influence emotions in a positive manner, can encourage participation. Based on the physiological measurements and qualitative data, Mandalynth tracing had an effect on the participants' anxiety. The participants' answers to the final interview questions show that a majority believed Mandalynth tracing to be helpful, particularly with relaxation and calming. Though the results of this study cannot support Mandalynths as 
a CAM, these effects echo the utility of mindfulness techniques of other CAMs $[17,20]$.

\section{Limitations and Suggestions for Future Research}

There are a few limitations which need to be considered in this study. The small sample size limits confidence in the statistical analysis of the quantitative and qualitative data. One participant experienced psychosis during the research, hampering the generalizability of the data. However, the small sample size allowed for a more in-depth analysis of the participants' individual experiences. This was also a short, time-limited study with only four tracing sessions. Longer-term effects of the intervention could be analyzed and could have encouraged more reflection of its benefits. Finally, on certain occasions, the location in which the research was conducted had excessive amounts of noise.

While the interview questions provided valuable qualitative data, they were possibly too vague. The interview questions were kept intentionally broad so as not to be leading. If the study were to be repeated, questions would be formulated to be less abstract and more direct to address the focus of the study. Questions could also have been administered before each tracing session, as this may have provided useful information about fluctuations in perceived level of anxiety during each session.

The findings suggest further research is needed on Mandalynth tracing as a mindfulness-based tool and its efficacy on participation in individuals with mental illnesses. To address this, future studies could incorporate Mandalyth tracing into a full-time residential program. For example, Mandalynth tracing could be utilized before each group therapy session and the perception of participation in the group session could be tracked. More consistent use over a longer period of time, particularly in the focused environment of a group therapy session, would likely yield more meaningful data.

\section{Conclusion}

Anxiety, as a common symptom of mental illness, has the power to influence self-perception and participation in activities $[3,7]$. Many of the participants stated it was more difficult to engage in activities when experiencing higher levels of anxiety and stress. The majority of participants felt their mood was positively affected by Mandalynth tracing. They reported feelings of relaxation, focus, centeredness, and having a "blank mind." This suggests Mandalynth tracing, as a mindfulness-based focusing tool, can be used as another type of coping strategy in stressful or anxiety-producing situations depending on individual preferences and current psychological symptoms.

Occupational therapists focus on empowering individuals to find ways of participating in activities which are meaningful to them Mandalynth tracing may lead to an increase in focus and a decrease in anxiety, which may cause an overall increase in an individual's ability to participate in meaningful occupations. The results of this study suggest Mandalynth tracing could be incorporated into OT practice as either a preparatory activity or as a coping strategy clients could take home and incorporate into their daily lives.

\section{Acknowledgement}

The authors would like to express their gratitude to Park Center for their participation and the six willing participants for their excitement and support throughout the study. They would also like to thank and Erin Redo for her advice and her generous donation of Mandalynths to this research effort.

\section{Conflicts of Interest}

No conflict of interest.

\section{References}

1. (WHO) World Health Organization (2014) Mental health: A state of wellbeing.

2. (NAMI) National Alliance on Mental Illness (2013) Mental health by the numbers.

3. Achim AM, Maziade M, Raymond É, Olivier D, Mérette C, et al. (2011) How prevalent are anxiety disorders in schizophrenia? A meta-analysis and critical review on a significant association. Schizophr Bull 37(4): 811-821.

4. Bajor LA, Gunzler D, Einstadter D, Thomas C, McCormick R, et al. (2015) Associations between comorbid anxiety, diabetes control, and overall medical burden in patients with serious mental illness and diabetes. Int J Psychiatry Med 49(4): 309-320.

5. Schneiderman N, Ironson G, Siegel SD (2005) Stress and health: Psychological, behavioral, and biological determinants. Annu Rev Clin Psychol 1: 607-628

6. American Occupational Therapy Association (2012) Occupational therapy's role in mental health promotion, prevention, \& intervention with children \& youth anxiety disorders.

7. Graydon MM, Linkenauger SA, Teachman BA, Proffitt DR (2012) Scared stiff: The influence of anxiety on the perception of action capabilities. Cogn Emo 26(7): 1301-1315.

8. Honda K, Jacobson JS (2005) Use of complementary and alternative medicine among United States adults: The influences of personality, coping strategies, and social support. Prev Med 40(1): 46-53.

9. American Occupational Therapy Association (2014) Occupational therapy practice framework: domain and process. American Journal of Occupational Therapy 68(1): S1-S48.

10. Denise R, Janine F, Canan O (2013) Exploring mindfulness with occupational therapists practicing in mental health contexts. Occupational Therapy in Mental Health 29(3): 279-292.

11. Tabish SA (2008) Complementary and alternative healthcare: Is it evidence-based? Int J Health Sci (Qassim) 2(1): V-IX.

12. Giese T (2003) Complementary and alternative medicine (CAM) position paper. Am J Occup Ther 59(6): 653-655.

13. Epstein RM (2003) Mindful practice in action (I): Technical competence, evidence-based medicine, and relationship-centered care. Families Systems and Health 21(1): 1-9.

14. Kabat Zinn J, Ludwig DS (2008) Mindfulness in medicine. JAMA 300(11): 1350-1352.

15. Brown LF, Davis LW, La Rocco VA, Strasburger A (2010) Participant perspectives on mindfulness meditation training for anxiety in schizophrenia. American Journal of Psychiatric Rehabilitation 13(3): 224-242.

16. Baer RA (2003) Mindfulness training as a clinical intervention: A conceptual and empirical review. Clinical Psychology: Science and Practice 10: 125-143.

17. Carmody J, Baer R (2008) Relationships between mindfulness practice and levels of mindfulness, medical and psychological symptoms and well-being in a mindfulness-based stress reduction program. J Behav Med 31(1): 23-33. 
18. Shapiro SL, Oman D, Thoresen CE, Plante TG, Flinders T (2008) Cultivating mindfulness: Effects on well-being. J Clin Psychol 64(7): 840-862.

19. Sokmen YC, Watters A (2016) Emotional regulation with mindfulness arts activities using a personalized self-soothing kit. Occupational Therapy in Mental Health 32(4): 345-369.

20. Joyce A, Etty Leal J, Zazryn T, Hamilton A, Hassed C (2010) Exploring a mindfulness meditation program on the mental health of upper primary children: A pilot study. Advances in School Mental Health Promotion $3(2): 17-25$

21. Park Center Nashville (2016) Park center: Recovery from mental illness.

22. Beck AT, Epstein N, Brown G, Steer RA (1988) An inventory for measuring clinical anxiety: Psychometric properties. Journal of Consulting and Clinical Psychology (56)6: 893-897.

23. American Heart Association (2017) Managing stress to control high blood pressure. 